\title{
$\theta$-type Calderón-Zygmund Operators and Commutators in Variable Exponents Herz space
}

https://doi.org/10.1515/math-2018-0133

Received July 4, 2018; accepted November 17, 2018

Abstract: The aim of this paper is to deal with the boundedness of the $\theta$-type Calderón-Zygmund operators and their commutators on Herz spaces with two variable exponents $p(\cdot), q(\cdot)$. It is proved that the $\theta$-type Calderón-Zygmund operators are bounded on the homogeneous Herz space with variable exponents $\dot{K}_{p(\cdot)}^{\alpha, q \cdot \cdot}\left(\mathbb{R}^{n}\right)$. Furthermore, the boundedness of the corresponding commutators generated by BMO function and Lipschitz function is also obtained respectively.

Keywords: $\theta$-type Calderón-Zygmund operator; commutator; Herz spaces with variable exponent; BMO space MSC: 42B20, 42B25, 42B35

\section{Introduction and Main Results}

The theory of Calderón-Zygmund operators, which has played very important roles in modern harmonic analysis with lots of extensive applications in the others fields of mathematics, has been extensively studied (see [1-6], for instance). In 1985, Yabuta introduced certain $\theta$-type Calderón-Zygmund operators to facilitate his study of certain classes of pseudodifferential operators (see [7]). Following the terminology of Yabuta, we recall the so-called $\theta$-type Calderón-Zygmund operators. Let $\theta$ be a non-negative and non-decreasing function on $\mathbb{R}^{+}=(0, \infty)$ satisfying

$$
\int_{0}^{1} \frac{\theta(t)}{t} \mathrm{~d} t<\infty .
$$

A measurable function $K(\cdot, \cdot)$ on $\mathbb{R}^{n} \times \mathbb{R}^{n} \backslash\left\{(x, x): x \in \mathbb{R}^{n}\right\}$ is said to be a $\theta$-type Calderón-Zygmund kernel if it satisfies

$$
|K(x, y)| \leq C|x-y|^{-n}, x=y,
$$

and

$$
\left|K(x, y)-K\left(x^{\prime}, y\right)\right|+\left|K(y, x)-K\left(y, x^{\prime}\right)\right| \leq C \theta\left(\frac{\left|x-x^{\prime}\right|}{|x-y|}\right)|x-y|^{-n}, \text { as }|x-y| \geq 2\left|x-x^{\prime}\right|, x=y .
$$

Definition 1.1 ${ }^{[7]}$ Let $T_{\theta}$ be a linear operator from $\mathcal{S}$ into its dual $\mathcal{S}^{\prime}$. One can say that $T_{\theta}$ is a $\theta$-type CalderónZygmund operator if it satisfies the following conditions:

(1) $T_{\theta}$ can be extended to be a bounded linear operator on $L^{2}\left(\mathbb{R}^{n}\right)$;

\footnotetext{
${ }^{\star}$ Corresponding Author: Shuangping Tao: College of Mathematics and Statistics, Northwest Normal University, Lanzhou 730070,Gansu, China, E-mail: taosp@nwnu.edu.cn

Yanqi Yang: College of Mathematics and Statistics, Northwest Normal University, Lanzhou 730070,Gansu, China,

E-mail: 18709498755@126.com
} 
(2) there is a $\theta$-type Calderón-Zygmund kernel $K(x, y)$ such that

$$
T_{\theta} f(x):=\int_{\mathbb{R}^{n}} K(x, y) f(y) \mathrm{d} y \text {, as } f \in C_{c}^{\infty}\left(\mathbb{R}^{n}\right) \text { and } x \notin \operatorname{supp} f .
$$

It is easy to see that the classical Calderón-Zygmund operator with standard kernel is a special case of $\theta$ type operator $T_{\theta}$ as $\theta(t)=t^{\delta}$ with $0<\delta \leq 1$. Given a locally integrable function $b$, the commutator generated by $T_{\theta}$ and $b$ is defined by

$$
\left[b, T_{\theta}\right] f(x)=b(x) T_{\theta} f(x)-T_{\theta}(b \cdot f)(x)=\int_{\mathbb{R}^{n}}[b(x)-b(y)] K(x, y) f(y) \mathrm{d} y .
$$

Such type of operators are extensively applied in PDE with non-smooth area. Many authors concentrate on the boundedness of these operators on various function spaces, we refer the reader to see [8-14] for its developments and applications. In [11], Quek-Yang established the boundedness of $T_{\theta}$ on spaces such as weighted Lebesgue spaces, weighted weak Lebesgue spaces, weighted Hardy spaces and weighted weak Hardy spaces. Ri-Zhang obtained the bounedness of $T_{\theta}$ on Hardy spaces with non-doubling measures and non-homogeneous metric measure spaces in $[12,13]$. Wang proved the boundedness of $T_{\theta}$ and $\left[b, T_{\theta}\right]$ on the generalized weighted Morrey spaces in [14]. Inspired by the results mentioned previously, a natural and interesting problem is to consider whether or not the $\theta$-type Calderón-Zygmund operators $T_{\theta}$ and their commutators $\left[b, T_{\theta}\right]$ are bounded on Herz space with variable exponents. The purpose of this paper is to give a positive answer.

The spaces with variable exponent have been widely studied in recent ten years. The results show that they are not only the generalized forms of the classical function spaces with invariable exponent, but also there are some new breakthroughs in the research techniques. These new real variable methods help people further understand the function spaces. Lebesgue spaces with variable exponent $L^{p(\cdot)}\left(\mathbb{R}^{n}\right)$ become one class of important function spaces due to the fundamental paper [15] by Kovóčik Rákosník. The theory of the function spaces with variable exponent have been applied in fluid dynamics, elastlcity dynamics, calculus of variations and differential equations with non-standard growth conditions(for example, see [16-20]). In [21], authors proved the extrapolation theorem which leads the boundedness of some classical operators including the commutators on $L^{p(\cdot)}\left(\mathbb{R}^{n}\right)$. Karlovich and Lerner also obtained the bundedness of the singular integral commutators in [22]. The boundedness of some typical operators is being studied with keen interest on spaces with variable exponent (see [23-26]). Recently, Tao and Yang established the boundedness of $\theta$-Type C-Z operators and their commutators generated respectively by BMO functions, Lipschitz functions and Besov functions on variable exponent Lebesgue spaces $L^{p(\cdot)}\left(\mathbb{R}^{n}\right)$ (see [27]), at the same time, the boundedness of singular integrals with variable kernel and fractional differentiations is also obtained.

It is well known that Herz spaces play an important role in harmonic analysis. After the Herz spaces with one exponent $p(\cdot)$ were introduced in [28], the boundedness of some operators and some characterizations of these spaces were studied widely (see [29-32]). In this paper, we will study the boundedness of the $\theta$-type Calderón-Zygmund operators $T_{\theta}$ and their commutators $\left[b, T_{\theta}\right]$ on Herz spaces with two variable exponents $p(\cdot), q(\cdot)$. In order to do this, we need to recall some notations and definitions.

Denote $\mathcal{P}\left(\mathbb{R}^{n}\right)$ to be the set of the all measurable functions $p(x)$ with $p_{-}=$: ess $\inf _{x \in \mathbb{R}^{n}} p(x)>1$ and $p_{+}=$: ess $\sup _{x \in \mathbb{R}^{n}} p(x)<\infty$ and $\mathcal{B}\left(\mathbb{R}^{n}\right)$ to be the set of all functions $p(\cdot) \in \mathcal{P}\left(\mathbb{R}^{n}\right)$ satisfying the condition that the HardyLittlewood maximal operator $M$ is bounded on $L^{p(\cdot)}\left(\mathbb{R}^{n}\right), \mathcal{P}^{0}\left(\mathbb{R}^{n}\right)$ the set of all measurable functions $p(x)$ with $p_{-}>0$ and $p_{+}<\infty$.

Given a function $p(x) \in \mathcal{P}\left(\mathbb{R}^{n}\right)$, the space $L^{p(x)}\left(\mathbb{R}^{n}\right)$ is now defined by ${ }^{[15]}$

$$
\|f\|_{L^{p(\cdot)}\left(\mathbb{R}^{n}\right)}=\inf \left\{\eta>0: \int_{\mathbb{R}^{n}}\left(\frac{|f(x)|}{\eta}\right)^{p(x)} \mathrm{d} x \leq 1\right\} .
$$

Given a function $p(\cdot) \in \mathcal{P}^{0}\left(\mathbb{R}^{n}\right)$, the space $L^{p(\cdot)}\left(\mathbb{R}^{n}\right)$ is defined by

$$
L^{p(\cdot)}\left(\mathbb{R}^{n}\right)=\left\{f:|f|^{p_{0}} \in L^{q(\cdot)}\left(\mathbb{R}^{n}\right) \text { for some } p_{0} \text { with } 0<p_{0}<p_{-} \text {and } q(x)=\frac{p(x)}{p_{0}}\right\} .
$$


It is easy to see that the above Luxemburg-Nakano quasi-norm is also equivalent with ${ }^{[21]}$

$$
\|f\|_{L^{p \cdot(\cdot)}\left(\mathbb{R}^{n}\right)}=\left\||f|^{p_{0}}\right\|_{L^{q(\cdot)}\left(\mathbb{R}^{n}\right)}^{1 / p_{0}},
$$

where $q(x)=\frac{p(x)}{p_{0}}$ and for some $p_{0}$ with $0<p_{0}<p_{-}$.

Before recalling the Herz spaces with two variable exponents, we will introduce the following function space, which is named as the mixed Lebesgue sequence space (see [33]).

Definition 1.2 ${ }^{[24,33]}$ Let $p(\cdot), q(\cdot) \in \mathcal{P}^{0}\left(\mathbb{R}^{n}\right)$. The mixed Lebesgue sequence space with variable exponent $l^{q(\cdot)}\left(L^{p(\cdot)}\right)$ is the collection of all sequences $\left\{f_{j}\right\}_{j=0}^{\infty}$ of measurable functions on $\mathbb{R}^{n}$ such that

$$
\left\|\left\{f_{j}\right\}_{j=0}^{\infty}\right\|_{l^{q(\cdot)}\left(L^{p(\cdot))}\right.}=\inf \left\{\mu>0: \Lambda_{l^{q \cdot(\cdot)}\left(L^{p \cdot(\cdot)}\right)}\left(\left\{f_{j} / \mu\right\}_{j=0}^{\infty}\right) \leq 1\right\}<\infty
$$

where

$$
\Lambda_{l^{q(\cdot)}\left(L^{p \cdot(\cdot)}\right)}\left(\left\{f_{j}\right\}_{j=0}^{\infty}\right)=\sum_{j=0}^{\infty} \inf \left\{\mu_{j}>0: \int_{\mathbb{R}^{n}}\left(\left|f_{j}(x)\right| / \mu_{j}^{\frac{1}{q(x)}}\right)^{p(x)} \mathrm{d} x \leq 1\right\} .
$$

Noting $q_{+}<\infty$, we see that

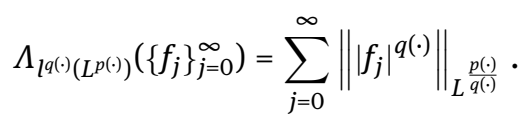

Let $k \in \mathbb{Z}, B_{k}=\left\{x \in \mathbb{R}^{n}:|x| \leq 2^{k}\right\}, C_{k}=B_{k} \backslash B_{k-1}, \chi_{k}=\chi_{C_{k}}$.

Definition 1.3 ${ }^{[24]}$ Let $\alpha \in \mathbb{R}, p(\cdot), q(\cdot) \in \mathcal{P}^{0}\left(\mathbb{R}^{n}\right)$. The homogeneous Herz space with variable exponent $\dot{K}_{p(\cdot)}^{\alpha, q(\cdot)}\left(\mathbb{R}^{n}\right)$ is defined by

$$
\dot{K}_{p(\cdot)}^{\alpha, q(\cdot)}\left(\mathbb{R}^{n}\right)=\left\{f \in L_{\mathrm{loc}}^{p(\cdot)}\left(\mathbb{R}^{n} \backslash\{0\}\right):\|f\|_{\dot{K}_{p(\cdot)}^{\alpha, q(\cdot)}\left(\mathbb{R}^{n}\right)}<\infty\right\},
$$

where

$$
\|f\|_{\dot{K}_{p(\cdot)}^{\alpha, q(\cdot)}\left(\mathbb{R}^{n}\right)}=\left\|\left\{2^{k \alpha}\left|f \chi_{k}\right|\right\}_{k=-\infty}^{\infty}\right\|_{l^{q(\cdot)}\left(L^{p(\cdot)}\right)}=\inf \left\{\eta>0, \sum_{k=-\infty}^{\infty}\left\|\left(2^{k \alpha}\left|f \chi_{k}\right| / \eta\right)^{q(\cdot)}\right\|_{L^{\frac{p(\cdot)}{q(\cdot)}}} \leq 1\right\} .
$$

Remark 1.1 (1) It is easy to see that $\dot{K}_{p(\cdot)}^{0, p(\cdot)}\left(\mathbb{R}^{n}\right)=L^{p(\cdot)}\left(\mathbb{R}^{n}\right)$ and if $p(x)=p_{0}$ and $q(x)=q_{0}$ are constants, then $\dot{K}_{p(\cdot)}^{\alpha, q(\cdot)}\left(\mathbb{R}^{n}\right)=\dot{K}_{p_{0}}^{\alpha, q_{0}}\left(\mathbb{R}^{n}\right)$ is just the usual Herz spaces (see [34]).

(2) If $q_{1}(\cdot), q_{2}(\cdot) \in \mathcal{P}^{0}\left(\mathbb{R}^{n}\right)$ satisfying $\left(q_{1}\right)_{+} \leq\left(q_{2}\right)_{-}$, then (see [24])

$$
\dot{K}_{p(\cdot)}^{\alpha, q_{1}(\cdot)}\left(\mathbb{R}^{n}\right) \subset \dot{K}_{p(\cdot)}^{\alpha, q_{2}(\cdot)}\left(\mathbb{R}^{n}\right) .
$$

Definition 1.5 ${ }^{[35]}$ When $0<\beta \leq 1$, the Homogeneous Lipschitz space $\operatorname{Lip}_{\beta}\left(\mathbb{R}^{n}\right)$ is the space of functions such that

$$
\|f\|_{\operatorname{Lip}_{\beta}}=\sup _{x, h \in \mathbb{R}^{n} ; h=0} \frac{|f(x+h)-f(x)|}{|h|^{\beta}}<\infty .
$$

Our main results in this paper are fomulated as follows.

Theorem 1.1 Suppose that $T_{\theta}$ is a $\theta$-type Calderón-Zygmund operators with $\theta$ satisfies $(1,1), p(\cdot) \in \mathcal{B}\left(\mathbb{R}^{n}\right)$, $q_{1}(\cdot), q_{2}(\cdot) \in \mathcal{P}^{0}\left(\mathbb{R}^{n}\right)$ with $\left(q_{2}\right)_{-} \geq\left(q_{1}\right)_{+}$and $p(\cdot) / q_{2}(\cdot) \in \mathcal{P}\left(\mathbb{R}^{n}\right)$. If $-n \delta_{12}<\alpha<n \delta_{11}$, where $\delta_{11}, \delta_{12}$ are the constants in Lemma 2.2, then the operator $T_{\theta}$ is bounded from $\dot{K}_{p(\cdot)}^{\alpha, q_{1}(\cdot)}\left(\mathbb{R}^{n}\right)$ to $\dot{K}_{p(\cdot)}^{\alpha, q_{2}(\cdot)}\left(\mathbb{R}^{n}\right)$.

Theorem 1.2 Let $b \in \mathrm{BMO}, m \in \mathbb{N}$ and $p(\cdot), q_{1}(\cdot), q_{2}(\cdot)$ and $\alpha$ are the same as in Theorem 1.1. Suppose that $\left[b, T_{\theta}\right]$ is defined by (1.5) with $\theta$ satisfying

$$
\int_{0}^{1} \frac{\theta(t)}{t}|\log t| \mathrm{d} t<\infty
$$


Then the commutator $\left[b, T_{\theta}\right]$ is bounded from $\dot{K}_{p(\cdot)}^{\alpha, q_{1}(\cdot)}\left(\mathbb{R}^{n}\right)$ to $\dot{K}_{p(\cdot)}^{\alpha, q_{2}(\cdot)}\left(\mathbb{R}^{n}\right)$.

Theorem 1.3 Let $b \in \operatorname{Lip}_{\beta}\left(\mathbb{R}^{n}\right)(0<\beta<1)$ and $\left[b, T_{\theta}\right]$ be defined by (1.5) with $\theta$ satisfying (1.6). Suppose that $q_{1}(\cdot), q_{2}(\cdot) \in \mathcal{P}^{0}\left(\mathbb{R}^{n}\right)$ with $\left(q_{2}\right)_{-} \geq\left(q_{1}\right)_{+}$and $p_{1}(\cdot), p_{2}(\cdot) \in \mathcal{B}\left(\mathbb{R}^{n}\right)$ is such that $\left(p_{1}\right)_{+}<\frac{n}{\beta}, 1 / p_{1}(x)-1 / p_{2}(x)=$ $\beta / n,(1-\beta / n) p_{2}(\cdot) \in \mathcal{B}\left(\mathbb{R}^{n}\right)$ and $p_{2}(\cdot) / q_{2}(\cdot) \in \mathcal{P}\left(\mathbb{R}^{n}\right)$. If $-n \delta_{12}<\alpha<n \delta_{11}$, then the commutator $\left[b, T_{\theta}\right]$ is bounded from $\dot{K}_{p_{1}(\cdot)}^{\alpha, q_{1}(\cdot)}\left(\mathbb{R}^{n}\right)$ to $\dot{K}_{p_{2}(\cdot)}^{\alpha, q_{2} \cdot \cdot(}\left(\mathbb{R}^{n}\right)$.

We make some conventions. In what follows, $C$ always denotes a positive constant which is independent of the main parameters involved but whose value may differ in different occurrences, $|E|$ denotes the Lebesgue measure of $E \in \mathbb{R}^{n}$. Given a function $f$, we denote the mean value of $f$ on $E$ by $f_{E}=: \frac{1}{|E|} \int_{E} f(x) \mathrm{d} x \cdot p^{\prime}(\cdot)$ means the conjugate exponent of $p(\cdot)$, namely, $1 / p(x)+1 / p^{\prime}(x)=1$ holds.

\section{Preliminary Lemmas}

Before proving the main results, we need the following lemmas.

Lemma 2.1 ${ }^{[15]}$ (Generalized Hölder's Inequality) Let $p(\cdot), p_{1}(\cdot), p_{2}(\cdot) \in p(\cdot) \in \mathcal{P}\left(\mathbb{R}^{n}\right)$.

(1) For any $f \in L^{p(\cdot)}\left(\mathbb{R}^{n}\right)$ and $g \in L^{p^{\prime}(\cdot)}\left(\mathbb{R}^{n}\right)$,

$$
\int_{\mathbb{R}^{n}}|f(x) g(x)| \mathrm{d} x \leq C_{p}\|f\|_{L^{p(\cdot)}}\|g\|_{L^{p^{\prime}(\cdot)}},
$$

where $C_{p}=1+1 / p^{-}-1 / p^{+}$.

(2) For any $f \in L^{p_{1}(\cdot)}\left(\mathbb{R}^{n}\right)$ and $g \in L^{p_{2}^{\prime}(\cdot)}\left(\mathbb{R}^{n}\right)$, when $1 / p(x)=1 / p_{1}(x)+1 / p_{2}(x)$, we have

$$
\|f(x) g(x)\|_{L^{p \cdot()\left(\mathbb{R}^{n}\right)}} \leq C_{p_{1}, p_{2}}\|f\|_{L^{p_{1}(\cdot)}}\|g\|_{L^{p_{2}^{(\cdot)}}},
$$

where $C_{p_{1}, p_{2}}=\left(1+1 / p_{1-}-1 / p_{1^{+}}\right)^{1 / p_{-}}$

Lemma 2.2 ${ }^{[22]}$ If $p_{i} \in \mathcal{B}\left(\mathbb{R}^{n}\right)(i=1,2)$, then there exist constants $p_{i, 1}, p_{i, 2}, C>0$, such that for all balls $B \in \mathbb{R}^{n}$ and all measurable subsets $S \in B$,

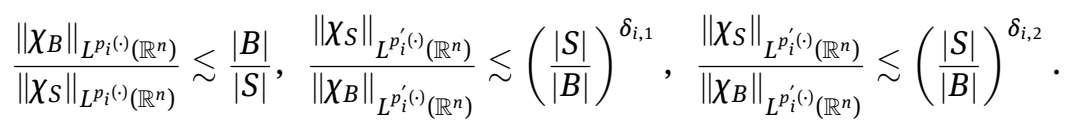

Lemma 2.3 ${ }^{[30]}$ If $p_{i}(\cdot) \in \mathcal{B}\left(\mathbb{R}^{n}\right)$, then there exists a constant $C>0$ such that for all balls $B \in \mathbb{R}^{n}$,

$$
\frac{1}{|B|}\left\|\chi_{B}\right\|_{L^{p(\cdot)\left(\mathbb{R}^{n}\right)}}\left\|\chi_{B}\right\|_{L^{p^{\prime}(\cdot)\left(\mathbb{R}^{n}\right)}} \leq C .
$$

Lemma $2.4^{[24]}$ Let $p(\cdot), q_{1}(\cdot) \in \mathcal{P}\left(\mathbb{R}^{n}\right)$. If $f \in L^{p(\cdot) q(\cdot)}\left(\mathbb{R}^{n}\right)$, then

$$
\min \left(\|f\|_{L^{p(\cdot) q(\cdot)}}^{q_{+}}\|f\|_{L^{p(\cdot) q(\cdot)}}^{q_{-}}\right) \leq\left\||f|^{q(\cdot)}\right\|_{L^{p(\cdot)}} \leq \max \left(\|f\|_{L^{p(\cdot)(\cdot)}}^{q_{+}},\|f\|_{L^{p(\cdot)(\cdot)}}^{q_{-}}\right) .
$$

Lemma $2.5^{[30]}$ Let $b \in \mathrm{BMO}$ and $m$ be a positive integer. There exists a constant $C>0$, such that for any $k, j \in \mathbb{Z}$ with $k>j$,

(1) $C^{-1}\|b\|_{\star}^{m} \leq \sup _{B} \frac{1}{\left\|\chi_{B}\right\|_{L^{p \cdot(\cdot)\left(\mathbb{R}^{n}\right)}}}\left\|\left(b-b_{B}\right)^{m} \chi_{B}\right\|_{L^{p \cdot(\cdot)\left(\mathbb{R}^{n}\right)}} \leq C\|b\|_{\star}^{m} ;$

(2) $\left\|\left(b-b_{B_{j}}\right)^{m} \chi_{B_{k}}\right\|_{L^{p \cdot(\cdot)}\left(\mathbb{R}^{n}\right)} \leq C(k-j)^{m}\|b\|_{\star}^{m}\left\|\chi_{B_{k}}\right\|_{L^{p \cdot(\cdot)}\left(\mathbb{R}^{n}\right)}$.

Lemma 2.6 ${ }^{[36]}$ If $p(\cdot) \in \mathcal{B}\left(\mathbb{R}^{n}\right)$, then there exist constants $0<\delta<1, C>0$, such that for all $Y \in \mathcal{T}$, all nonnegative numbers $t_{Q}$ and all $f \in L_{\text {loc }}^{1}\left(\mathbb{R}^{n}\right)$ with $f_{Q}=0(Q \in Y)$,

$$
\left\|\sum_{Q \in Y} t_{Q}\left|\frac{f}{f_{Q}}\right|^{\delta} \chi_{Q}\right\|_{L^{p(\cdot)\left(\mathbb{R}^{n}\right)}} \leq C\left\|\sum_{Q \in Y} t_{Q} X_{Q}\right\|_{L^{p \cdot(\cdot)}\left(\mathbb{R}^{n}\right)},
$$


where $\mathcal{T}$ denotes all families of disjoint and open cube in $\mathbb{R}^{n}$.

Lemma 2.7 ${ }^{[24]}$ Let $b \in \operatorname{Lip}_{\beta}\left(\mathbb{R}^{n}\right)$ and $m$ be a positive integer. There exists a constant $C>0$, such that for any $k, j \in \mathbb{Z}$ with $k>j$

(1) $C^{-1}\|b\|_{\operatorname{Lip}_{\beta}}^{m} \leq \frac{1}{|B|^{m \beta / n}}\left\|\chi_{B}\right\|_{L^{p(\cdot)}\left(\mathbb{R}^{n}\right)}^{-1}\left\|\left(b-b_{B}\right)^{m} \chi_{B}\right\|_{L^{p \cdot(\cdot)}\left(\mathbb{R}^{n}\right)} \leq C\|b\|_{\operatorname{Lip}_{\beta}}^{m} ;$

(2) $\left\|\left(b-b_{B_{j}}\right)^{m} \chi_{B_{k}}\right\|_{L^{p(\cdot)}\left(\mathbb{R}^{n)}\right.} \leq C\left|B_{k}\right|^{m \beta / n}\|b\|_{\operatorname{Lip}_{\beta}}^{m}\left\|\chi_{B}\right\|_{L^{p(\cdot)}\left(\mathbb{R}^{n}\right)}$.

Lemma 2.8 ${ }^{[37]}$ Let $f \in \operatorname{Lip}_{\beta}\left(\mathbb{R}^{n}\right), 0<\beta<1,1 \leq p<\infty, B_{1} \subset B_{2}$. We have

(1) $\|f\|_{\operatorname{Lip}_{\beta}} \approx \sup _{B} \frac{1}{|B|^{\beta / n}}\left(\frac{1}{|B|} \int_{B}\left|f(x)-f_{B}\right|^{p} \mathrm{~d} x\right)^{1 / p}$;

(2) $\left|f_{B_{1}}-f_{B_{2}}\right| \leq C\|f\|_{\operatorname{Lip}_{\beta}}\left|B_{2}\right|^{\beta / n}$.

Lemma 2.9 ${ }^{[24]}$ Let $k \in \mathbb{N}, a_{k} \geq 0,1 \leq p_{k}<\infty$. Then

$$
\sum_{k=0}^{\infty} a_{k}^{p_{k}} \leq\left(\sum_{k=0}^{\infty} a_{k}\right)^{p_{*}}, \text { where } p_{*}=\left\{\begin{array}{l}
\min _{k \in \mathbb{N}} p_{k}, \sum_{k=0}^{\infty} a_{k} \leq 1 ; \\
\max _{k \in \mathbb{N}} p_{k}, \sum_{k=0}^{\infty} a_{k}>1 .
\end{array}\right.
$$

Lemma 2.10 ${ }^{[27]}$ Suppose that $T_{\theta}$ is a $\theta$-type Calderón-Zygmund operator and $\theta$ satisfies $(1,1)$. Let $p(\cdot) \in \mathcal{B}\left(\mathbb{R}^{n}\right)$, then there exits a constant $C$ independent of $f$ such that

$$
\left\|T_{\theta}(f)\right\|_{L^{p(\cdot)}\left(\mathbb{R}^{n}\right)} \leq C\|f\|_{L^{p(\cdot)}\left(\mathbb{R}^{n}\right)} \cdot
$$

Lemma 2.11 ${ }^{[27]}$ Let $b \in \operatorname{BMO}\left(\mathbb{R}^{n}\right)$. Suppose that $p(\cdot) \in \mathcal{B}\left(\mathbb{R}^{n}\right)$ and $\theta$ satisfies (1.6), then there exists a constant $C$ independent of $f$ such that

$$
\left\|\left[b, T_{\theta}\right](f)\right\|_{L^{p \cdot(\cdot)\left(\mathbb{R}^{n}\right)}} \leq C\|b\|\left\|_{\star}\right\| f \|_{L^{p(\cdot)}\left(\mathbb{R}^{n}\right)} .
$$

Lemma 2.12 ${ }^{[27]}$ Let $b \in \operatorname{Lip}_{\beta}\left(\mathbb{R}^{n}\right)$ for $0<\beta<1$. Suppose that $\theta$ satisfies $(1,1)$ and $p(\cdot) \in \mathcal{P}\left(\mathbb{R}^{n}\right)$ be such that $p_{+}<\frac{n}{\beta}$. Define $q(\cdot)$ by

$$
\frac{1}{p(x)}-\frac{1}{q(x)}=\frac{\beta}{n} .
$$

If $q(\cdot)\left(1-\frac{\beta}{n}\right) \in \mathcal{B}\left(\mathbb{R}^{n}\right)$, then there exists a constant $C$ independent of $f$ such that

$$
\left\|\left[b, T_{\theta}\right](f)\right\|_{L^{q(\cdot)}\left(\mathbb{R}^{n}\right)} \leq C\|b\|_{L_{i p_{\beta}\left(\mathbb{R}^{n}\right)}\|f\|_{L^{p(\cdot)}\left(\mathbb{R}^{n}\right)} .}
$$

\section{Proof of Main Theorems}

Proof of Theorem 1.1. Let $f \in \dot{K}_{p(\cdot)}^{\alpha, q_{1}(\cdot)}\left(\mathbb{R}^{n}\right)$. Write

$$
f(x)=\sum_{j=-\infty}^{\infty} f(x) \chi_{j}(x) \triangleq \sum_{j=-\infty}^{\infty} f_{j}(x) .
$$

Due to $p(\cdot) / q_{2}(\cdot) \in \mathcal{P}\left(\mathbb{R}^{n}\right)$, which implies $\inf \left(p(\cdot) / q_{2}(\cdot)\right) \geq 1$, then we have

$$
\left\|T_{\theta}(f)\right\|_{\dot{K}_{p(\cdot)}^{\alpha, q_{2}(\cdot)}\left(\mathbb{R}^{n)}\right.}=\inf \left\{\eta>0: \sum_{k=-\infty}^{\infty}\left\|\left(\frac{2^{k \alpha}\left|T_{\theta}(f) \chi_{k}\right|}{\eta}\right)^{q_{2}(\cdot)}\right\|_{\frac{p(\cdot)}{q^{q_{2} \cdot(\cdot)}}} \leq 1\right\} .
$$

Since

$$
\left\|\left(\frac{2^{k \alpha}\left|T_{\theta}(f) \chi_{k}\right|}{\eta}\right)^{q_{2}(\cdot)}\right\|_{L^{\frac{p(\cdot)}{q_{2} \cdot(\cdot)}}} \leq C\left\|\left(\frac{2^{k \alpha}\left|\sum_{j=-\infty}^{k-3} T_{\theta}\left(f_{j}\right) \chi_{k}\right|}{\eta_{11}}\right)^{q_{2}(\cdot)}\right\|_{L^{\frac{p(\cdot)}{q_{2}(\cdot)}}}
$$




$$
+C\left\|\left(\frac{2^{k \alpha}\left|\sum_{j=k-2}^{k+2} T_{\theta}\left(f_{j}\right) \chi_{k}\right|}{\eta_{12}}\right)^{q_{2}(\cdot)}\right\|_{\frac{p(\cdot)}{q_{2_{2} \cdot(\cdot)}}}+C\left\|\left(\frac{2^{k \alpha}\left|\sum_{j=k+3}^{\infty} T_{\theta}\left(f_{j}\right) \chi_{k}\right|}{\eta_{13}}\right)^{q_{2}(\cdot)}\right\|_{L^{\frac{p(\cdot)}{q_{2} \cdot(\cdot)}}},
$$

where

$$
\begin{gathered}
\eta_{11}=\left\|\left\{2^{k \alpha}\left|\sum_{j=-\infty}^{k-3} T_{\theta}\left(f_{j}\right) \chi_{k}\right|\right\}_{k=-\infty}^{\infty}\right\|\left\|_{l_{q_{2}(\cdot)\left(L^{p(\cdot)}\right)},}, \eta_{12}=\right\|\left\{2^{k \alpha}\left|\sum_{j=k-2}^{k+2} T_{\theta}\left(f_{j}\right) \chi_{k}\right|\right\}_{k=-\infty}^{\infty} \|_{l_{q_{2}(\cdot)\left(L^{p \cdot(\cdot)}\right)}}, \\
\eta_{13}=\left\|\left\{2^{k \alpha}\left|\sum_{j=k+3}^{\infty} T_{\theta}\left(f_{j}\right) \chi_{k}\right|\right\}_{k=-\infty}^{\infty}\right\|_{l_{q_{2}(\cdot)\left(L^{p(\cdot)}\right)} \text { and } \eta=\eta_{11}+\eta_{12}+\eta_{13} .}
\end{gathered}
$$

Hence,

$$
\left\|T_{\theta}(f)\right\|_{\dot{K}_{p(\cdot)}^{\alpha, q_{2}(\cdot)}\left(\mathbb{R}^{n)}\right.} \leq C\left(\eta_{11}+\eta_{12}+\eta_{13}\right) .
$$

This implies that, in order to prove our theorem, we only need to show $\eta_{11}, \eta_{12}, \eta_{13} \leq C\|f\|_{\dot{K}_{p(\cdot)}^{\alpha, q_{1}(\cdot)}\left(\mathbb{R}^{n}\right)}$. For simplicity, we denote $\lambda_{0}=\|f\|_{\dot{K}_{p(\cdot)}^{\alpha, q_{1}(\cdot)}\left(\mathbb{R}^{n}\right)^{\text {. }}}$.

First, we consider $\eta_{12}$. By Lemma 2.4 and using Aoki-Rolewicz's theorem, we have

$$
\begin{aligned}
\sum_{k=-\infty}^{\infty}\left\|\left(\frac{2^{k \alpha}\left|\sum_{j=k-2}^{k+2} T_{\theta}\left(f_{j}\right) \chi_{k}\right|}{\lambda_{0}}\right)^{q_{2}(\cdot)}\right\|_{L^{\frac{p(\cdot)}{q_{2}(\cdot)}}} & \leq \sum_{k=-\infty}^{\infty}\left\|\frac{2^{k \alpha}\left|\sum_{j=k-2}^{k+2} T_{\theta}\left(f_{j}\right) \chi_{k}\right|}{\lambda_{0}}\right\|_{L^{p \cdot(\cdot)}}^{\left(q_{2}^{1}\right)_{k}} \\
& \leq C \sum_{k=-\infty}^{\infty}\left(\sum_{j=k-2}^{k+2}\left\|\frac{2^{k \alpha}\left|T_{\theta}\left(f_{j}\right) \chi_{k}\right|}{\lambda_{0}}\right\|_{L^{p \cdot(\cdot)}}\right)^{\left(q_{2}^{1}\right)_{k}},
\end{aligned}
$$

where

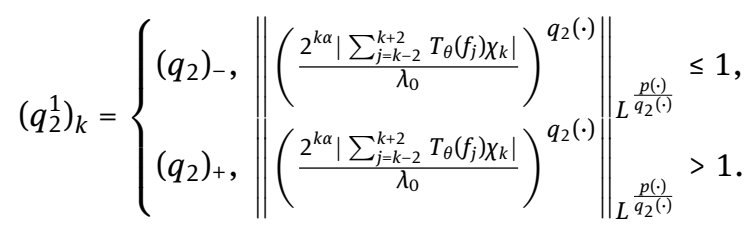

By using Lemma 2.10, it follows

$$
\begin{aligned}
\sum_{k=-\infty}^{\infty}\left\|\left(\frac{2^{k \alpha}\left|\sum_{j=k-2}^{k+2} T_{\theta}\left(f_{j}\right) \chi_{k}\right|}{\lambda_{0}}\right)^{q_{2}(\cdot)}\right\|_{L^{\frac{p(\cdot)}{q_{2}(\cdot)}}} & C C \sum_{k=-\infty}^{\infty}\left(\sum_{j=k-2}^{k+2}\left\|\frac{2^{k \alpha}\left|f_{j}\right|}{\lambda_{0}}\right\|_{L^{p \cdot(\cdot)}}\right)^{\left(q_{2}^{1}\right)_{k}} \\
& \leq C \sum_{k=-\infty}^{\infty}\left\|\frac{2^{k \alpha}\left|f \chi_{k}\right|}{\lambda_{0}}\right\|_{L^{p(\cdot)}}^{\left(q_{2}^{1}\right)_{k}} .
\end{aligned}
$$

Since $f \in \dot{K}_{p(\cdot)}^{\alpha, q_{1}(\cdot)}\left(\mathbb{R}^{n}\right)$, we can easily see $\left\|\frac{2^{k \alpha}\left|f x_{k}\right|}{\lambda_{0}}\right\|_{L^{p(\cdot)}} \leq 1$ and $\sum_{k=-\infty}^{\infty}\left\|\left(\frac{2^{k \alpha}\left|f x_{k}\right|}{\lambda_{0}}\right)^{q_{1}(\cdot)}\right\|_{L^{\frac{p(\cdot)}{q_{1} \cdot(\cdot)}}} \leq 1$. Hence, by Lemma 2.4 and Lemma 2.9, we have

$$
\begin{aligned}
& \sum_{k=-\infty}^{\infty}\left\|\left(\frac{2^{k \alpha}\left|\sum_{j=k-2}^{k+2} T_{\theta}\left(f_{j}\right) \chi_{k}\right|}{\lambda_{0}}\right)^{q_{2}(\cdot)}\right\|_{L^{\frac{p(\cdot)}{q_{2} \cdot(\cdot)}}} \leq C \sum_{k=-\infty}^{\infty}\left\|\left(\frac{2^{k \alpha}\left|f \chi_{k}\right|}{\lambda_{0}}\right)^{q_{1}(\cdot)}\right\|_{L^{\frac{p(\cdot)}{q_{1} \cdot(\cdot)}}}^{\frac{\left(q_{2}^{1}\right)_{k}}{\left.q_{1}\right)_{+}}} \\
& \leq C\left\{\sum_{k=-\infty}^{\infty}\left\|\left(\frac{2^{k \alpha}\left|f \chi_{k}\right|}{\lambda_{0}}\right)^{q_{1}(\cdot)}\right\|_{L^{\frac{p(\cdot)}{q_{1} \cdot(\cdot)}}}\right\}^{q_{*}} \\
& \leq C \text {. }
\end{aligned}
$$


Here $\left(q_{1}\right)_{+} \leq\left(q_{2}\right)_{-} \leq\left(q_{2}^{1}\right)_{k}$ and $q_{\star}=\min _{k \in \mathbb{Z}} \frac{\left(q_{2}^{1}\right)_{k}}{\left(q_{1}\right)_{+}}$. Consequently, we have $\eta_{12} \leq C \lambda_{0}$.

Now let us turn to estimate $\eta_{11}$.

$$
\left|T_{\theta}\left(f_{j}\right)(x)\right|=\left|\int_{\mathbb{R}^{n}} K(x, y) f_{j}(y) \mathrm{d} y\right| \leq C \int_{\mathbb{R}^{n}} \frac{1}{|x-y|^{n}}\left|f_{j}(y)\right| \mathrm{d} y .
$$

Let $x \in C_{k}, y \in C_{j}, j \leq k-3$, then we have $|x-y| \geq|x|-|y| \geq \frac{3}{8}|x|$. Hence, we have

$$
\left|T_{\theta}\left(f_{j}\right)(x)\right| \leq C|x|^{-n}\left\|f_{j}\right\|_{L^{1}\left(\mathbb{R}^{n}\right)} .
$$

Thus, By Lemmas 2.1-2.4 and the fact that $\left\|\left(\frac{2^{j \alpha}\left|f x_{j}\right|}{\lambda_{0}}\right)^{q_{1}(\cdot)}\right\|_{L^{p(\cdot) q_{1}(\cdot)}} \leq 1$, it follows that

$$
\begin{aligned}
& \sum_{k=-\infty}^{\infty}\left\|\left(\frac{2^{k \alpha}\left|\sum_{j=-\infty}^{k-3} T_{\theta}\left(f_{j}\right) \chi_{k}\right|}{\lambda_{0}}\right)^{q_{2}(\cdot)}\right\|_{L^{\frac{p(\cdot)}{q_{2}(\cdot)}}} \leq C \sum_{k=-\infty}^{\infty}\left\|\left(\frac{\left.2^{k \alpha}\left|\sum_{j=-\infty}^{k-3}\right| x\right|^{-n}\left\|f_{j}\right\|_{L^{1}\left(\mathbb{R}^{n}\right)} \chi_{k} \mid}{\lambda_{0}}\right)^{q_{2}(\cdot)}\right\|_{L^{\frac{p(\cdot)}{q_{2}(\cdot)}}} \\
& \leq C \sum_{k=-\infty}^{\infty}\left\|\frac{\left.2^{k \alpha}\left|\sum_{j=-\infty}^{k-3}\right| x\right|^{-n}\left\|f_{j}\right\|_{L^{1}\left(\mathbb{R}^{n}\right)} \chi_{k} \mid}{\lambda_{0}}\right\|_{L^{p(\cdot)}}^{\left(q_{2}^{2}\right)_{k}} \\
& \leq C \sum_{k=-\infty}^{\infty}\left(2^{k(\alpha-n)} \sum_{j=-\infty}^{k-3}\left\|\frac{f_{j}}{\lambda_{0}}\right\|_{L^{p}\left(\mathbb{R}_{n}\right)}\left\|\chi_{j}\right\|_{L^{p^{(\cdot)}}}\left\|\chi_{k}\right\|_{L^{p(\cdot)}}\right)^{\left(q_{2}^{2}\right)_{k}} \\
& \leq C \sum_{k=-\infty}^{\infty}\left(2^{k(\alpha-n)} \sum_{j=-\infty}^{k-3}\left\|\frac{f_{j}}{\lambda_{0}}\right\|_{L^{p}\left(\mathbb{R}_{n}\right)} \frac{\left\|\chi_{B_{k}}\right\|_{L^{p^{\prime}(\cdot)\left(\mathbb{R}^{n}\right)}}}{\left\|\chi_{B_{j}}\right\|_{L^{p^{(\cdot)}\left(\mathbb{R}^{n}\right)}}}\left|B_{k}\right|\right)^{\left(q_{2}^{2}\right)_{k}} \\
& \leq C \sum_{k=-\infty}^{\infty}\left\{\sum_{j=-\infty}^{k-3} 2^{(k-j)\left(\alpha-n \delta_{11}\right)}\left\|\left(\frac{\left|2^{j \alpha} f \chi_{j}\right|}{\lambda_{0}}\right)^{q_{1}(\cdot)}\right\|_{\left.L^{(\cdot) \cdot q_{1} \cdot(\cdot)} \mathbb{R}^{n}\right)}^{\frac{1}{\left(q_{1}\right)_{+}}}\right\}^{\left(q_{2}^{2}\right)_{k}},
\end{aligned}
$$

where

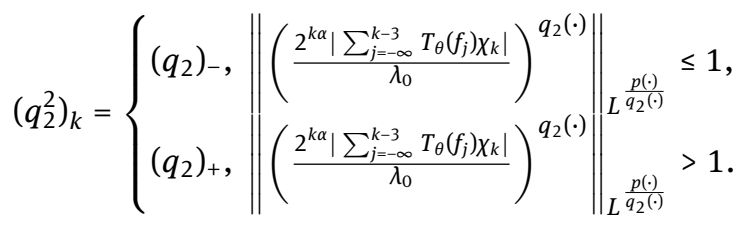

If $\left(q_{1}\right)_{+}<1$, then by Lemma 2.9 and the fact $\left(q_{1}\right)_{+} \leq\left(q_{2}\right)_{-} \leq\left(q_{2}^{1}\right)_{k}$, we have

$$
\begin{aligned}
& \sum_{k=-\infty}^{\infty}\left\|\left(\frac{2^{k \alpha}\left|\sum_{j=-\infty}^{k-3} T_{\theta}\left(f_{j}\right) \chi_{k}\right|}{\lambda_{0}}\right)^{q_{2}(\cdot)}\right\|_{\frac{p(\cdot)}{q_{q_{2} \cdot(\cdot)}}} \leq C \sum_{k=-\infty}^{\infty}\left\{\sum_{j=-\infty}^{k-3} 2^{(k-j)\left(\alpha-n \delta_{11}\right)}\left\|\left(\frac{\left|2^{j \alpha} f \chi_{j}\right|}{\lambda_{0}}\right)^{q_{1}(\cdot)}\right\|_{L^{p(\cdot) q_{1}(\cdot)\left(\mathbb{R}^{n}\right)}}\right\}^{\frac{\left(q_{2}^{2}\right)_{k}}{\left(q_{1}\right)_{+}}} \\
& \leq C\left\{\sum_{j=-\infty}^{\infty}\left\|\left(\frac{\left|2^{j \alpha} f \chi_{j}\right|}{\lambda_{0}}\right)^{q_{1}(\cdot)}\right\|_{L^{p(\cdot) q_{1} \cdot(\cdot)\left(\mathbb{R}^{n}\right)}} \sum_{k=j+3}^{\infty} 2^{(k-j)\left(\alpha-n \delta_{11}\right)}\right\}^{q_{*}} \leq C,
\end{aligned}
$$

where $q_{\star}=\min _{k \in \mathbb{Z}} \frac{\left(q_{2}^{2}\right)_{k}}{\left(q_{1}\right)_{+}}$.

If $\left(q_{1}\right)_{+} \geq 1$, then $1 \leq\left(q_{1}\right)_{+} \leq\left(q_{2}\right)_{-} \leq\left(q_{2}^{1}\right)_{k}$. Thus, for $\alpha<n \delta_{11}$, by applying Hölder's inequality and Lemma 2.9, we get

$$
\sum_{k=-\infty}^{\infty}\left\|\left(\frac{2^{k \alpha}\left|\sum_{j=-\infty}^{k-3} T_{\theta}\left(f_{j}\right) \chi_{k}\right|}{\lambda_{0}}\right)^{q_{2}(\cdot)}\right\|_{\frac{p(\cdot)}{q_{2} \cdot(\cdot)}} \leq C \sum_{k=-\infty}^{\infty}\left\{\sum_{j=-\infty}^{k-3} 2^{(k-j)\left(\alpha-n \delta_{11}\right)\left(q_{1}\right)_{+} / 2}\left\|\left(\frac{\left|2^{j \alpha} f \chi_{j}\right|}{\lambda_{0}}\right)^{q_{1}(\cdot)}\right\|_{L^{p(\cdot) q_{1}(\cdot)\left(\mathbb{R}^{n}\right)}}\right\}^{\frac{\left(q_{2}^{2}\right)_{k}}{\left(q_{1}\right)_{+}}}
$$




$$
\begin{aligned}
& \times\left(\sum_{j=-\infty}^{k-3} 2^{(k-j)\left(\alpha-n \delta_{11}\right)\left(\left(q_{1}\right)_{+}\right)^{\prime} / 2}\right)^{\frac{\left(q_{2}^{2}\right)_{k}}{\left(q_{1}\right)^{\prime}}} \\
\leq & C\left\{\sum_{j=-\infty}^{\infty}\left\|\left(\frac{\left|2^{j \alpha} f \chi_{j}\right|}{\lambda_{0}}\right)^{q_{1}(\cdot)}\right\|_{\left.L^{p(\cdot) q_{1} \cdot(\cdot)} \mathbb{R}^{n}\right)} \sum_{k=j+3}^{\infty} 2^{(k-j)\left(\alpha-n \delta_{11}\right)\left(q_{1}\right)_{+} / 2}\right\}^{q_{*}} \leq C,
\end{aligned}
$$

where $q_{\star}=\min _{k \in \mathbb{Z}} \frac{\left(q_{2}^{2}\right)_{k}}{\left(q_{1}\right)_{+}}$. This implies that $\eta_{21} \leq C \lambda_{0}$.

Finally, we are going to estimate $\eta_{23}$. Let $x \in C_{k}, y \in C_{j}, j \geq k+3$, then we have $|x-y| \geq|y|-|x| \geq \frac{3}{8}|y|$. Hence, we have

$$
\left|T_{\theta}\left(f_{j}\right)(x)\right| \leq C 2^{-j n}\left\|f_{j}\right\|_{L^{1}\left(\mathbb{R}^{n}\right)} .
$$

Thus, By Lemmas 2.1-2.4 and the fact that $\left\|\left(\frac{2^{j \alpha}\left|f x_{j}\right|}{\lambda_{0}}\right)^{q_{1}(\cdot)}\right\|_{L^{p(\cdot) q_{1}(\cdot)}} \leq 1$, it follows that

$$
\begin{aligned}
& \sum_{k=-\infty}^{\infty}\left\|\left(\frac{2^{k \alpha}\left|\sum_{j=k+3}^{\infty} T_{\theta}\left(f_{j}\right) \chi_{k}\right|}{\lambda_{0}}\right)^{q_{2}(\cdot)}\right\|_{L^{\frac{p(\cdot)}{q_{2}(\cdot)}}} \leq C \sum_{k=-\infty}^{\infty}\left\|\left(\frac{2^{k \alpha}\left|\sum_{j=k+3}^{\infty} 2^{-j n}\left\|f_{j}\right\|_{L^{1}\left(\mathbb{R}^{n}\right)} X_{k}\right|}{\lambda_{0}}\right)^{q_{2}(\cdot)}\right\|_{L^{\frac{p(\cdot)}{q_{2}(\cdot)}}} \\
& \leq C \sum_{k=-\infty}^{\infty}\left(2^{k \alpha} \sum_{j=k+3}^{\infty} 2^{-j n}\left\|\frac{f_{j}}{\lambda_{0}}\right\|_{L^{p}\left(\mathbb{R}_{n}\right)}\left\|\chi_{j}\right\|_{L^{p^{\prime} \cdot(\cdot)}}\left\|\chi_{k}\right\|_{L^{p \cdot \cdot}}\right)^{\left(q_{2}^{3}\right)_{k}} \\
& \leq C \sum_{k=-\infty}^{\infty}\left(2^{k \alpha} \sum_{j=k+3}^{\infty} 2^{-j n}\left\|\frac{f \chi_{j}}{\lambda_{0}}\right\|_{L^{p}\left(\mathbb{R}_{n}\right)} \frac{\left\|\chi_{B_{k}}\right\|_{L^{p(\cdot)}\left(\mathbb{R}^{n}\right)}}{\left\|\chi_{B_{j}}\right\|_{L^{p \cdot()}\left(\mathbb{R}^{n}\right)}}\left|B_{j}\right|\right)^{\left(q_{2}^{3}\right)_{k}} \\
& \leq C \sum_{k=-\infty}^{\infty}\left\{\sum_{j=k+3}^{\infty} 2^{(k-j)\left(\alpha+n \delta_{12}\right)}\left\|\left(\frac{\left|2^{j \alpha} f \chi_{j}\right|}{\lambda_{0}}\right)^{q_{1}(\cdot)}\right\|_{\left.L^{(\cdot) \cdot q_{1} \cdot(\cdot)} \mathbb{R}^{n}\right)}^{\frac{1}{\left(q_{1}\right)_{+}}}\right\}^{\left(q_{2}^{3}\right)_{k}},
\end{aligned}
$$

where

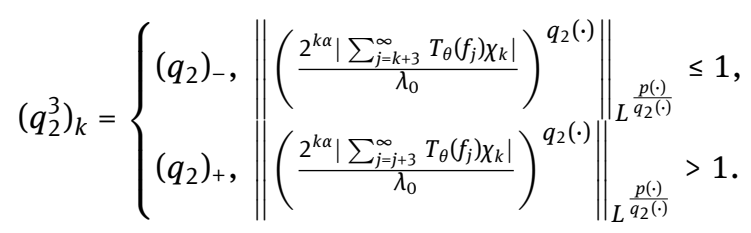

Noticing that $\left(q_{2}\right)_{-} \geq\left(q_{1}\right)_{+}$and $\alpha>-n \delta_{12}$, by a similar argument about $\eta_{11}$, we have $\eta_{13} \leq C \lambda_{0}$.

Combing the estimates of $\eta_{11}, \eta_{12}$ and $\eta_{13}$, we finish the proof of Theorem 1.1.

Proof of Theorem 1.2. Let $b \in \mathrm{BMO}\left(\mathbb{R}^{n}\right), f \in \dot{K}_{p(\cdot)}^{\alpha, q_{1}(\cdot)}\left(\mathbb{R}^{n}\right)$. As in the proof of Theorem 1.1, we write

$$
f(x)=\sum_{j=-\infty}^{\infty} f(x) \chi_{j}(x) \triangleq \sum_{j=-\infty}^{\infty} f_{j}(x) .
$$

Since

$$
\begin{aligned}
\left\|\left(\frac{2^{k \alpha}\left|\left[b, T_{\theta}\right](f) \chi_{k}\right|}{\eta}\right)^{q_{2}(\cdot)}\right\|_{L^{\frac{p(\cdot)}{q_{2} \cdot(\cdot)}}} & C\left\|\left(\frac{2^{k \alpha}\left|\sum_{j=-\infty}^{k-3}\left[b, T_{\theta}\right]\left(f_{j}\right) \chi_{k}\right|}{\eta_{21}}\right)^{q_{2}(\cdot)}\right\|_{L^{\frac{p(\cdot)}{q_{2}(\cdot)}}} \\
& +C\left\|\left(\frac{2^{k \alpha}\left|\sum_{j=k-2}^{k+2}\left[b, T_{\theta}\right]\left(f_{j}\right) \chi_{k}\right|}{\eta_{22}}\right)^{q_{2}(\cdot)}\right\|_{L^{\frac{p(\cdot)}{q_{2}(\cdot)}}} \| \\
& +C\left\|\left(\frac{2^{k \alpha}\left|\sum_{j=k+3}^{\infty}\left[b, T_{\theta}\right]\left(f_{j}\right) \chi_{k}\right|}{\eta_{23}}\right)^{q_{2}(\cdot)}\right\|_{L^{\frac{p(\cdot)}{q_{2} \cdot(\cdot)}}},
\end{aligned}
$$


where

$$
\begin{gathered}
\eta_{21}=\left\|\left\{2^{k \alpha}\left|\sum_{j=-\infty}^{k-3}\left[b, T_{\theta}\right]\left(f_{j}\right) \chi_{k}\right|\right\}_{k=-\infty}^{\infty}\right\|_{l^{q_{2} \cdot(\cdot)}\left(L^{p \cdot(\cdot)}\right)}, \eta_{22}=\left\|\left\{2^{k \alpha}\left|\sum_{j=k-2}^{k+2}\left[b, T_{\theta}\right]\left(f_{j}\right) \chi_{k}\right|\right\}_{k=-\infty}^{\infty}\right\|_{l_{q_{2}(\cdot)}\left(L^{p \cdot(\cdot)}\right)}, \\
\eta_{23}=\left\|\left\{2^{k \alpha}\left|\sum_{j=k+3}^{\infty}\left[b, T_{\theta}\right]\left(f_{j}\right) \chi_{k}\right|\right\}_{k=-\infty}^{\infty}\right\|_{\left.q_{q_{2}(\cdot)} L^{p(\cdot)}\right)} \text { and } \eta=\eta_{21}+\eta_{22}+\eta_{23} .
\end{gathered}
$$

Hence, it is enough to prove $\eta_{21}, \eta_{22}, \eta_{23} \leq C\|b\|_{\star} \lambda_{0}$, where $\lambda_{0}=\|f\|_{\dot{K}_{p(\cdot)}^{\alpha, q_{1} \cdot(\cdot)}}\left(\mathbb{R}^{n}\right)^{\text {. }}$

We first estimate $\eta_{22}$. Noticing that $\left[b, T_{\theta}\right]$ is bounded on $L^{p(\cdot)}\left(\mathbb{R}^{n}\right)$ (see Lemma 2.11), as the same argument about in $\eta_{12}$ in the proof of Theorem 1.1, we immediately get

$$
\sum_{k=-\infty}^{\infty}\left\|\left(\frac{2^{k \alpha}\left|\sum_{j=k-2}^{k+2}\left[b, T_{\theta}\right]\left(f_{j}\right) \chi_{k}\right|}{\lambda_{0}\|b\|_{\star}}\right)^{q_{2}(\cdot)}\right\|_{L^{\frac{p(\cdot)}{q_{2} \cdot(\cdot)}}} \leq C .
$$

That is to say, $\eta_{22} \leq C\|b\| \|_{\star} \lambda_{0}$.

Next, we estimate $\eta_{21}$. Let $x \in C_{k}, j \leq k-3, \operatorname{supp} f_{j} \in C_{j}$. By the estimation of $T_{\theta}\left(f_{j}\right)$ in the proof of Theorem 1.1, we have

$$
\left|T_{\theta}\left(f_{j}\right)(x)\right| \leq C|x|^{-n}\left\|f_{j}\right\|_{L^{1}\left(\mathbb{R}^{n}\right)} .
$$

Then, it follows that

$$
\left|\left[b, T_{\theta}\right] f_{j}(x)\right|=\left|T_{\theta}\left[(b(x)-b) f_{j}\right](x)\right| \leq C|x|^{-n}\left\|(b(\cdot)-b) f_{j}\right\|_{L^{1}\left(\mathbb{R}^{n}\right)} .
$$

Thus, using Lemma 2.4 and Aoki-Rolewicz's theorem, we obtain

$$
\begin{aligned}
& \sum_{k=-\infty}^{\infty}\left\|\left(\frac{2^{k \alpha}\left|\sum_{j=-\infty}^{k-3}\left[b, T_{\theta}\right]\left(f_{j}\right) \chi_{k}\right|}{\lambda_{0}\|b\|_{\star}}\right)^{q_{2}(\cdot)}\right\|_{L^{\frac{p \cdot(\cdot)}{q_{2} \cdot(\cdot)}}} \\
& \leq C \sum_{k=-\infty}^{\infty}\left\|\left(\frac{\left.2^{k \alpha}\left|\sum_{j=-\infty}^{k-3}\right| x\right|^{-n}\left\|(b(\cdot)-b) f_{j}\right\|_{L^{1}\left(\mathbb{R}^{n}\right)} X_{k} \mid}{\lambda_{0}\|b\|_{\star}}\right)^{q_{2}(\cdot)}\right\|_{L^{\frac{p(\cdot)}{q_{2} \cdot(\cdot)}}} \\
& \leq C \sum_{k=-\infty}^{\infty}\left\|\frac{\left.2^{k \alpha}\left|\sum_{j=-\infty}^{k-3}\right| x\right|^{-n}\left\|(b(\cdot)-b) f_{j}\right\|_{L^{1}\left(\mathbb{R}^{n}\right)} X_{k} \mid}{\lambda_{0}\|b\|_{\star}}\right\|_{L^{p}(\cdot)}^{\left(q_{2}^{2}\right)_{k}} \\
& \leq C \sum_{k=-\infty}^{\infty}\left(2^{k(\alpha-n)} \sum_{j=-\infty}^{k-3}\left\|\frac{\left|\left(b-b_{j}\right) f_{j}\right|}{\lambda_{0}\|b\|_{\star}}\right\|_{L^{1}\left(\mathbb{R}_{n}\right)}\left\|\chi_{k}\right\|_{L^{p(\cdot)}}\right)^{\left(q_{2}^{2}\right)_{k}} \\
& \leq C \sum_{k=-\infty}^{\infty}\left(2^{k(\alpha-n)} \sum_{j=-\infty}^{k-3}\left\|\frac{\left|f_{j}\right|}{\lambda_{0}}\right\|_{L^{p}\left(\mathbb{R}_{n}\right)} \frac{1}{\|b\|_{\star}}\left\|\left(b-b_{j}\right) \chi_{B_{j}}\right\|_{\left.L^{p^{p^{\prime} \cdot}}\right)}\left\|\chi_{k}\right\|_{L^{p(\cdot)}}\right)^{\left(q_{2}^{2}\right)_{k}},
\end{aligned}
$$

where $b_{j}=b_{C_{j}}=\frac{1}{\left|C_{j}\right|} \int_{C_{j}} b(y) \mathrm{d} y$ and

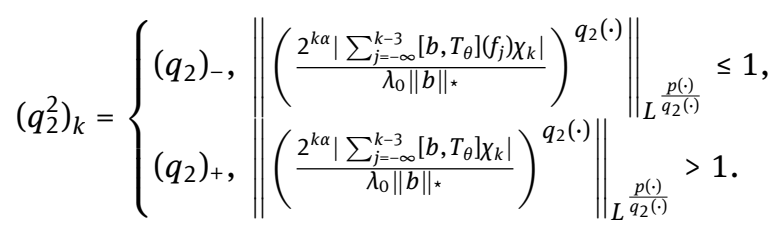

Applying the Hölder's inequality and Lemma 2.5 with the case $m=1$, we know that 


$$
\begin{aligned}
& \sum_{k=-\infty}^{\infty}\left\|\left(\frac{2^{k \alpha}\left|\sum_{j=-\infty}^{k-3}\left[b, T_{\theta}\right]\left(f_{j}\right) \chi_{k}\right|}{\lambda_{0}\|b\|_{\star}}\right)^{q_{2}(\cdot)}\right\|_{L^{\frac{p(\cdot)}{q_{2}(\cdot)}}} \\
& \leq C \sum_{k=-\infty}^{\infty}\left(2^{k(\alpha-n)} \sum_{j=-\infty}^{k-3}\left\|\frac{\left|f_{j}\right|}{\lambda_{0}}\right\|_{L^{p}\left(\mathbb{R}_{n}\right)} \frac{1}{\|b\|_{\star}}\left\|\left(b-b_{j}\right) \chi_{B_{j}}\right\|_{L^{p^{\prime} \cdot(\cdot)}}\left\|\chi_{k}\right\|_{L^{p(\cdot)}}\right)^{\left(q_{2}^{2}\right)_{k}} \\
& \leq C \sum_{k=-\infty}^{\infty}\left(2^{k(\alpha-n)} \sum_{j=-\infty}^{k-3}\left\|\frac{\left|f_{j}\right|}{\lambda_{0}}\right\|_{L^{p}\left(\mathbb{R}_{n}\right)}\left\|\chi_{j}\right\|_{L^{p^{\prime}(\cdot)}}(k-j)\left\|\chi_{B_{k}}\right\|_{L^{p(\cdot)}}\right)^{\left(q_{2}^{2}\right)_{k}} \\
& \leq C \sum_{k=-\infty}^{\infty}\left(2^{k(\alpha-n)} \sum_{j=-\infty}^{k-3}(k-j)\left|B_{k}\right| \frac{\left\|\chi_{B_{j}}\right\|_{L^{p^{\prime}(\cdot)}}}{\left\|\chi_{B_{k}}\right\|_{L^{p^{\prime}(\cdot)}}}\left\|\frac{\left|f_{j}\right|}{\lambda_{0}}\right\|_{L^{p}\left(\mathbb{R}_{n}\right)}\right)^{\left(q_{2}^{2}\right)_{k}}
\end{aligned}
$$

Furthermore, by the same argument as $\eta_{11}$ in the proof of Theorem 1.1, we have

$$
\begin{aligned}
& \sum_{k=-\infty}^{\infty}\left\|\left(\frac{2^{k \alpha}\left|\sum_{j=-\infty}^{k-3}\left[b, T_{\theta}\right]\left(f_{j}\right) \chi_{k}\right|}{\lambda_{0}\|b\|_{\star}}\right)^{q_{2}(\cdot)}\right\|_{L^{\frac{p(\cdot)}{q_{2}(\cdot)}}} \\
& \leq C \sum_{k=-\infty}^{\infty}\left(\sum_{j=-\infty}^{k-3}(k-j) 2^{(k-j)\left(\alpha-n \delta_{11}\right)}\left\|\left(\frac{\left|f_{\chi_{j}}\right|}{\lambda_{0}}\right)^{q_{1}(\cdot)}\right\|_{L^{p}\left(\mathbb{R}_{n}\right)}^{\frac{1}{\left(q_{1}\right)_{+}}}\right)^{\left(q_{2}^{2}\right)_{k}} . \\
& \leq C\left\{\begin{array}{l}
\left\{\sum_{j=-\infty}^{\infty}\left\|\left(\frac{\left|2^{j \alpha} f_{\chi_{j}}\right|}{\lambda_{0}}\right)^{q_{1}(\cdot)}\right\|_{L^{p(\cdot) q_{1}(\cdot)}} \sum_{k=j+3}^{\infty}(k-j) 2^{(k-j)\left(\alpha-n \delta_{11}\right)}\right\}^{q^{*}},(p)_{+} \leq 1, \\
\left\{\sum_{j=-\infty}^{\infty}\left\|\left(\frac{\left|2^{j \alpha} f_{\chi_{j}}\right|}{\lambda_{0}}\right)^{q_{1}(\cdot)}\right\|_{L^{p(\cdot) q_{1}(\cdot)}} \sum_{k=j+3}^{\infty}(k-j) 2^{(k-j)\left(\alpha-n \delta_{11}\right)\left(q_{1}\right)_{+} / 2}\right\}^{q^{*}},(p)_{+}>1 .
\end{array}\right.
\end{aligned}
$$

$\leq C$,

where $q_{\star}=\min _{k \in \mathbb{Z}} \frac{\left(q_{2}^{2}\right)_{k}}{\left(q_{1}\right)_{+}}$. Therefore, $\eta_{21} \leq C\|b\|{ }_{\star} \lambda_{0}$.

Finally, we estimate $\eta_{23}$. Let $x \in C_{k}, y \in C_{j}, j \geq k+3$, supp $f_{j} \in C_{j}$. By the estimation of the $T_{\theta}\left(f_{j}\right)$ in the proof of Theorem 1.1, we have

$$
\left|T_{\theta}\left(f_{j}\right)(x)\right| \leq C 2^{-j n}\left\|f_{j}\right\|_{L^{1}\left(\mathbb{R}^{n}\right)}
$$

From this, it follows

$$
\left|\left[b, T_{\theta}\right] f_{j}(x)\right|=\left|T_{\theta}\left[(b(x)-b) f_{j}\right](x)\right| \leq C 2^{-j n}\left\|(b(\cdot)-b) f_{j}\right\|_{L^{1}\left(\mathbb{R}^{n}\right)} \cdot
$$

Thus, when $\alpha>-n \delta_{12}$, as in the similar way to estimate $\eta_{21}$ before, we obtain

$$
\begin{aligned}
& \sum_{k=-\infty}^{\infty}\left\|\left(\frac{2^{k \alpha}\left|\sum_{j=k+3}^{\infty}\left[b, T_{\theta}\right]\left(f_{j}\right) \chi_{k}\right|}{\lambda_{0}\|b\|_{\star}}\right)^{q_{2}(\cdot)}\right\|_{L^{\frac{p(\cdot)}{q_{2}(\cdot)}}} \\
& \leq C \sum_{k=-\infty}^{\infty} \|\left.\frac{2^{k \alpha} \mid \sum_{j=k+3}^{\infty} 2^{-j n}\left\|(b(\cdot)-b) f_{j}\right\|_{L^{1}\left(\mathbb{R}^{n}\right) \chi_{k} \mid} \|_{L^{p}(\cdot)}}{\lambda_{0}\|b\|_{\star}}\right|_{\left.q_{2}^{3}\right)_{k}} ^{\left(q_{2}^{3}\right)_{k}} \\
& \leq C \sum_{k=-\infty}^{\infty}\left(2^{k \alpha} \sum_{j=k+3}^{\infty} 2^{j n}\left\|\frac{\left|(b(\cdot)-b) f_{j}\right|}{\lambda_{0}\|b\|_{\star}}\right\|_{L^{1}\left(\mathbb{R}_{n}\right)}\left\|\chi_{k}\right\|_{L^{p(\cdot)}}\right)^{(1)}
\end{aligned}
$$




$$
\begin{aligned}
& \leq C \sum_{k=-\infty}^{\infty}\left(2^{k \alpha} \sum_{j=-\infty}^{k-3} 2^{j n}\left\|\frac{\left|f_{j}\right|}{\lambda_{0}}\right\|_{L^{p}\left(\mathbb{R}_{n}\right)} \frac{1}{\|b\|_{*}}\left\|\left(b-b_{k}\right) \chi_{B_{j}}\right\|_{L^{p^{(\cdot)}}}\left\|\chi_{k}\right\|_{L^{p \cdot \cdot}}\right)^{\left(q_{2}^{3}\right)_{k}} \\
& \leq C \sum_{k=-\infty}^{\infty}\left(2^{k \alpha} \sum_{j=k+3}^{\infty} 2^{j n}(k-j)\left|B_{j}\right|\left\|\chi_{B_{k}}\right\|_{L^{p \cdot \cdot}}\left\|\chi_{B_{j}}\right\|_{L^{p(\cdot)}}\left\|\frac{\left|f_{j}\right|}{\lambda_{0}}\right\|_{L^{p}\left(\mathbb{R}_{n}\right)}\right)^{\left(q_{2}^{3}\right)_{k}} \\
& \leq C \sum_{k=-\infty}^{\infty}\left\{\sum_{j=k+3}^{\infty}(j-k) 2^{(k-j)\left(\alpha+n \delta_{12}\right)}\left\|\left(\frac{\left|f \chi_{j}\right|}{\lambda_{0}}\right)^{q_{1} \cdot(\cdot)}\right\|_{L^{p \cdot(\cdot)}\left(\mathbb{R}^{n}\right)}^{\frac{1}{\left(q_{1}\right)_{+}}}\right\}^{\left(q_{2}^{3}\right)_{k}} \leq C,
\end{aligned}
$$

where

$$
\left(q_{2}^{3}\right)_{k}=\left\{\begin{array}{l}
\left(q_{2}\right)_{-},\left\|\left(\frac{\left.2^{k \alpha} \mid \sum_{j=k+3}^{\infty}\left[b, T_{\theta}\right] f_{j}\right) \chi_{k} \mid}{\lambda_{0}\|b\|_{\star}}\right)^{q_{2}(\cdot)}\right\|_{\frac{p(\cdot)}{q_{2}}} \leq 1, \\
\left(q_{2}\right)_{+},\left\|\left(\frac{2^{k \alpha}\left|\sum_{j=k+3}^{\infty}\left[b, T_{\theta}\right] X_{k}\right|}{\lambda_{0}\|b\|_{\star}}\right)^{q_{2}(\cdot)}\right\|_{L^{\frac{p(\cdot)}{q_{2}(\cdot)}}}>1 .
\end{array}\right.
$$

Hence,

$$
\eta_{23} \leq C\|b\|_{\star} \lambda_{0} .
$$

Combing the estimates of $\eta_{21}, \eta_{22}$ and $\eta_{23}$, we finish the proof of Theorem 1.2.

Proof of Theorem 1.3. Let $b \in \operatorname{Lip}_{\beta}\left(\mathbb{R}^{n}\right), 0<\beta<1, f \in \dot{K}_{p_{1}(\cdot)}^{\alpha, q_{1}(\cdot)}\left(\mathbb{R}^{n}\right)$. Write

$$
f(x)=\sum_{j=-\infty}^{\infty} f(x) \chi_{j}(x) \triangleq \sum_{j=-\infty}^{\infty} f_{j}(x) .
$$

We use similar notations as in the proof of Theorem 1.2. Let

$$
\begin{gathered}
\eta_{31}=\left\|\left\{2^{k \alpha}\left|\sum_{j=-\infty}^{k-3}\left[b, T_{\theta}\right]\left(f_{j}\right) \chi_{k}\right|\right\}_{k=-\infty}^{\infty}\right\|_{l_{q_{2}(\cdot)}\left(L^{p_{2}(\cdot)}\right)}, \eta_{32}=\left\|\left\{2^{k \alpha}\left|\sum_{j=k-2}^{k+2}\left[b, T_{\theta}\right]\left(f_{j}\right) \chi_{k}\right|\right\}_{k=-\infty}^{\infty}\right\|_{l_{q_{2}(\cdot)}\left(L^{p_{2}(\cdot)}\right)}, \\
\eta_{33}=\left\|\left\{2^{k \alpha}\left|\sum_{j=k+3}^{\infty}\left[b, T_{\theta}\right]\left(f_{j}\right) \chi_{k}\right|\right\}_{k=-\infty}^{\infty}\right\|_{l_{q_{2} \cdot(\cdot)}\left(L^{p_{2}(\cdot)}\right)} \text { and } \eta=\eta_{31}+\eta_{32}+\eta_{33} .
\end{gathered}
$$

Similarly, we have

$$
\left\|\left[b, T_{\theta}\right](f)\right\|_{\dot{K}_{p_{2}(\cdot)}^{\alpha, q_{2} \cdot(\cdot)}\left(\mathbb{R}^{n}\right)} \leq C \eta=C\left(\eta_{31}+\eta_{32}+\eta_{33}\right) .
$$

We are now going to estimate $\eta_{31}, \eta_{32}, \eta_{33}$. For simplicity, we also denote $\left.\lambda_{0}=\|f\|_{\dot{K}_{p_{1}(\cdot)}^{\alpha, q_{1}(\cdot)}} \mathbb{R}^{n}\right)$.

We first estimate $\eta_{32}$. Noticing that $\left[b, T_{\theta}\right]$ is bounded $L^{p_{1}(\cdot)}\left(\mathbb{R}^{n}\right)$ to $L^{p_{2}(\cdot)}\left(\mathbb{R}^{n}\right)$ (Lemma 2.12), as the same argument about in $\eta_{12}$ in the proof of Theorem 1.1, we immediately get

$$
\sum_{k=-\infty}^{\infty}\left\|\left(\frac{2^{k \alpha}\left|\sum_{j=k-2}^{k+2}\left[b, T_{\theta}\right]\left(f_{j}\right) \chi_{k}\right|}{\lambda_{0}\|b\|_{\operatorname{Lip}_{\beta}\left(\mathbb{R}^{n}\right)}}\right)^{q_{2}(\cdot)}\right\|_{L^{\frac{p_{2}(\cdot)}{q_{2} \cdot \cdot}}} \leq C .
$$

Thus, $\eta_{32} \leq C\|b\|_{\operatorname{Lip}_{\beta}\left(\mathbb{R}^{n}\right)} \lambda_{0}$.

Now let us turn to estimate $\eta_{31}$. Let $x \in C_{k}, j \leq k-3$, supp $f_{j} \in C_{j}$. By the estimation of $\eta_{21}$ in the proof of Theorem 1.2 and the generalized Hölder's inequality, we have

$$
\sum_{k=-\infty}^{\infty}\left\|\left(\frac{2^{k \alpha}\left|\sum_{j=-\infty}^{k-3}\left[b, T_{\theta}\right]\left(f_{j}\right) \chi_{k}\right|}{\lambda_{0}\|b\|_{\operatorname{Lip}_{\beta}\left(\mathbb{R}^{n}\right)}}\right)^{q_{2}(\cdot)}\right\|_{L^{\frac{p_{2} \cdot(\cdot)}{q_{2} \cdot(\cdot)}}}
$$




$$
\begin{aligned}
& \leq C \sum_{k=-\infty}^{\infty}\left(2^{k(\alpha-n)} \sum_{j=-\infty}^{k-3}\left\|\frac{\left|\left(b-b_{j}\right) f_{j}\right|}{\lambda_{0}\|b\|_{\operatorname{Lip}_{\beta}\left(\mathbb{R}^{n}\right)}}\right\|_{L^{p_{1}\left(\mathbb{R}_{n}\right)}}\left\|\chi_{k}\right\|_{L^{p_{2}(\cdot)}}\right)^{\left(q_{2}^{2}\right)_{k}} \\
& \leq C \sum_{k=-\infty}^{\infty}\left(2^{k(\alpha-n)} \sum_{j=-\infty}^{k-3}\left\|\frac{f_{j}}{\lambda_{0}}\right\|_{L^{p_{1}\left(\mathbb{R}_{n}\right)}} \frac{\left\|\left(b-b_{j}\right) \chi_{B_{k}}\right\|_{L^{\left.p_{2} \cdot \cdot\right)}}}{\|b\|_{\operatorname{Lip}_{\beta}\left(\mathbb{R}^{n}\right)}}\right)^{\left(q_{2}^{2}\right)_{k}} \\
& \leq C \sum_{k=-\infty}^{\infty}\left(2^{k(\alpha-n)} \sum_{j=-\infty}^{k-3}\left\|\frac{\left|f_{j}\right|}{\lambda_{0}}\right\|_{L^{p_{1}\left(\mathbb{R}_{n}\right)}} \frac{1}{\|b\|_{\operatorname{Lip}_{\beta}\left(\mathbb{R}^{n}\right)}}\left\|\left(b-b_{j}\right) \chi_{B_{j}}\right\|_{L^{p_{1}^{\prime} \cdot(\cdot)}}\left\|\chi_{k}\right\|_{L^{\left.p_{2} \cdot \cdot \cdot \cdot\right)}}\right)^{\left(q_{2}^{2}\right)_{k}} \\
& \leq C \sum_{k=-\infty}^{\infty}\left(2^{k(\alpha-n)} \sum_{j=-\infty}^{k-3}\left\|\frac{\left|f_{j}\right|}{\lambda_{0}}\right\|_{L^{p_{1}\left(\mathbb{R}_{n}\right)}}\left\|\chi_{B_{k}}\right\|_{L^{p_{2}}(\cdot)} \frac{\left\|\left(b-b_{j}\right) \chi_{B_{j}}\right\|_{L^{p_{1}^{\prime}(\cdot)}}}{\|b\|_{\operatorname{Lip}_{\beta}\left(\mathbb{R}^{n}\right)}}\right)^{\left(q_{2}^{2}\right)_{k}},
\end{aligned}
$$

where

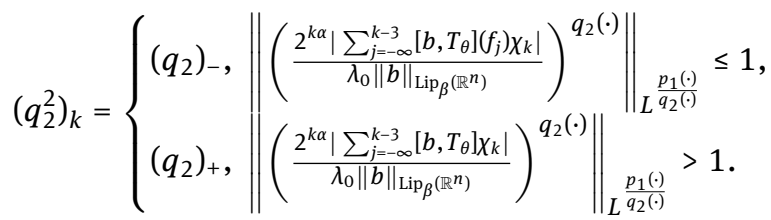

As $\frac{1}{p_{1}(x)}-\frac{1}{p_{2}(x)}=\frac{\beta}{n}$, we use the following fact (see [38])

$$
C_{1}|B|^{\beta / n}\left\|\chi_{B}\right\|_{L^{p_{2}(\cdot)}\left(\mathbb{R}^{n}\right)} \leq\left\|\chi_{B}\right\|_{L^{p_{1}(\cdot)}\left(\mathbb{R}^{n}\right)} \leq C_{2}|B|^{\beta / n}\left\|\chi_{B}\right\|_{L^{p_{2}(\cdot)}\left(\mathbb{R}^{n}\right)} .
$$

Together with Lemma 2.2-2.4 and Lemma 2.7, we obtain

$$
\begin{aligned}
& \sum_{k=-\infty}^{\infty}\left\|\left(\frac{2^{k \alpha}\left|\sum_{j=-\infty}^{k-3}\left[b, T_{\theta}\right]\left(f_{j}\right) \chi_{k}\right|}{\lambda_{0}\|b\|_{\operatorname{Lip}_{\beta}\left(\mathbb{R}^{n}\right)}}\right)^{q_{2}(\cdot)}\right\|_{L^{\frac{p_{2}(\cdot)}{q_{2}(\cdot)}}} \\
& \leq C \sum_{k=-\infty}^{\infty}\left(2^{k(\alpha-n)} \sum_{j=-\infty}^{k-3}\left\|\frac{\left|f_{j}\right|}{\lambda_{0}}\right\|_{L^{p_{1}\left(\mathbb{R}_{n}\right)}} \frac{\left|B_{j}\right|^{\beta / n}}{\left|B_{k}\right|^{\beta / n}}\left\|\chi_{B_{j}}\right\|_{L^{p_{1}^{\prime}(\cdot)}}\left\|\chi_{k}\right\|_{L^{p_{1}(\cdot)}}\right)^{\left(q_{2}^{2}\right)_{k}} \\
& \leq \sum_{k=-\infty}^{\infty}\left(2^{k(\alpha-n)} \sum_{j=-\infty}^{k-3}\left\|\frac{\left|f_{j}\right|}{\lambda_{0}}\right\|_{L^{p_{1}\left(\mathbb{R}_{n}\right)}}\left\|\chi_{B_{j}}\right\|_{L^{p_{1}^{\prime}(\cdot)}}\left\|\chi_{k}\right\|_{L^{p_{1}(\cdot)}}\right)^{\left(q_{2}^{2}\right)_{k}}
\end{aligned}
$$

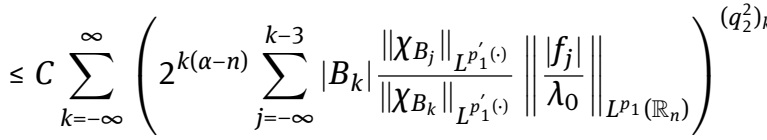

$$
\begin{aligned}
& \leq C \sum_{k=-\infty}^{\infty}\left(\sum_{j=-\infty}^{k-3} 2^{(k-j)\left(\alpha-n \delta_{11}\right)}\left\|\left(\frac{\left|f_{j}\right|}{\lambda_{0}}\right)^{q_{1}(\cdot)}\right\|_{L^{p_{1}\left(\mathbb{R}_{n}\right)}}^{\frac{1}{\left.q_{1}\right)_{+}}}\right)^{\left(q_{2}^{2}\right)_{k}} .
\end{aligned}
$$

Furthermore, by the same argument as $\eta_{11}$ in the proof of Theorem 1.1, we get

This implies that

$$
\sum_{k=-\infty}^{\infty}\left\|\left(\frac{2^{k \alpha}\left|\sum_{j=-\infty}^{k-3}\left[b, T_{\theta}\right]\left(f_{j}\right) \chi_{k}\right|}{\lambda_{0}\|b\|_{\operatorname{Lip}_{\beta}\left(\mathbb{R}^{n}\right)}}\right)^{q_{2}(\cdot)}\right\|_{L^{\frac{p_{1}(\cdot)}{q_{2}(\cdot)}}} \leq C .
$$

$$
\eta_{31} \leq C\|b\|_{\operatorname{Lip}_{\beta}\left(\mathbb{R}^{n}\right)} \lambda_{0} .
$$

Finally, we estimate $\eta_{33}$. Let $x \in C_{k}, y \in C_{j}, j \geq k+3$, supp $f_{j} \in C_{j}$. By the estimation of the $\eta_{23}$ in the proof of Theorem 1.2 and the generalized Hölder's inequality, we have

$$
\sum_{k=-\infty}^{\infty}\left\|\left(\frac{2^{k \alpha}\left|\sum_{j=k+3}^{\infty}\left[b, T_{\theta}\right]\left(f_{j}\right) \chi_{k}\right|}{\lambda_{0}}\right)^{q_{2}(\cdot)}\right\|_{L^{\frac{p_{2} \cdot(\cdot)}{q_{2} \cdot(\cdot)}}}
$$




$$
\begin{aligned}
& \leq C \sum_{k=-\infty}^{\infty}\left(2^{k \alpha} \sum_{j=k+3}^{\infty} 2^{-j n}\left\|\frac{\left|\left(b-b_{k}\right) f_{j}\right|}{\lambda_{0}\|b\|_{\operatorname{Lip}_{\beta}\left(\mathbb{R}^{n}\right)}}\right\|_{L^{1}\left(\mathbb{R}_{n}\right)}\left\|\chi_{k}\right\|_{L^{p_{2}(\cdot)}}\right)^{\left(q_{2}^{3}\right)_{k}} \\
& \leq C \sum_{k=-\infty}^{\infty}\left(2^{k(\alpha-n)} \sum_{j=k+3}^{\infty} 2^{-j n}\left\|\frac{\left|f_{j}\right|}{\lambda_{0}}\right\|_{L^{1}\left(\mathbb{R}_{n}\right)} \frac{\left\|\left(b-b_{k}\right) \chi_{B_{k}}\right\|_{L^{p_{2}(\cdot)}}}{\|b\|_{\operatorname{Lip}_{\beta}\left(\mathbb{R}^{n}\right)}^{\left(q_{2}^{3}\right)_{k}}}\right)^{,}
\end{aligned}
$$

where

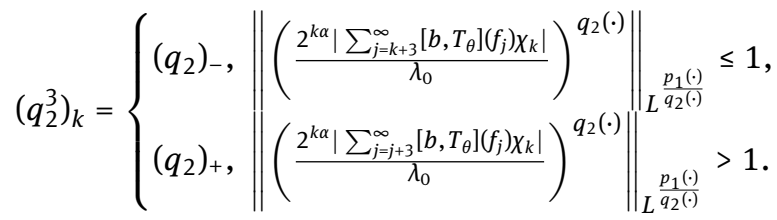

Observe that $1 / p_{1}(x)-1 / p_{2}(x)=\beta / n$, which implies $1 / p_{2}^{\prime}(x)-1 / p_{1}^{\prime}(x)=\beta / n$. Hence, when $\alpha>-n \delta_{22}$, as argued about $\eta_{31}$ before, one has

$$
\begin{aligned}
& \sum_{k=-\infty}^{\infty}\left\|\left(\frac{2^{k \alpha}\left|\sum_{j=k+3}^{\infty}\left[b, T_{\theta}\right]\left(f_{j}\right) \chi_{k}\right|}{\lambda_{0}}\right)^{q_{2}(\cdot)}\right\|_{L^{\frac{p_{2}(\cdot)}{q_{2} \cdot(\cdot)}}}
\end{aligned}
$$

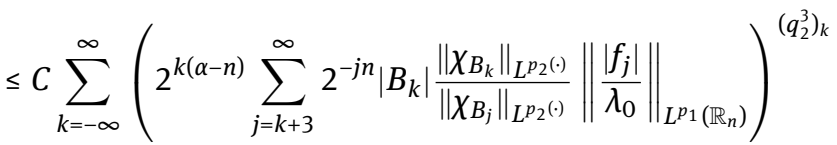

$$
\begin{aligned}
& \leq C \sum_{k=-\infty}^{\infty}\left(\sum_{j=k+3}^{\infty} 2^{(k-j)\left(\alpha+n \delta_{22}\right)}\left\|\left(\frac{\left|f \chi_{j}\right|}{\lambda_{0}}\right)^{q_{1}(\cdot)}\right\|_{L^{p_{1} \cdot(\cdot)}\left(\mathbb{R}_{n}\right)}^{\frac{1}{\left(q_{1}\right)_{+}}}\right)^{\left(q_{2}^{3}\right)_{k}} \leq C .
\end{aligned}
$$

Therefore,

$$
\eta_{33} \leq C\|b\|_{\operatorname{Lip}_{\beta}\left(\mathbb{R}^{n}\right)} \lambda_{0} .
$$

Summing up the estimates of $\eta_{31}, \eta_{32}$, and $\eta_{33}$, it follows that

$$
\left\|\left[b, T_{\theta}\right](f)\right\|_{\dot{K}_{p_{2}(\cdot)}^{\alpha, q_{2} \cdot(\cdot)}\left(\mathbb{R}^{n}\right)} \leq C\|b\|_{\operatorname{Lip}_{\beta}\left(\mathbb{R}^{n}\right)}\|f\|_{\dot{K}_{p_{1}(\cdot)}^{\alpha, q_{1} \cdot(\cdot)}\left(\mathbb{R}^{n}\right)} .
$$

This finishes the proof of Theorem 1.3.

Conflict of interest: The authors declare that there is no conflict of interests regarding the publication of this paper.

Authors' contributions: All authors contributed equally to the writing of this paper. All authors read and approved the final manuscript.

Acknowledgement: The authors would like to express their deep thanks to the referee for his/her very careful reading and many valuable comments and suggestions. This work is supported by National Natural Science Foundation of China (Grant No. 11561062).

\section{References}

[1] Calderón A., Zygmund A., On the existence of certain singular integrals, Acta Math., 1952, 88, 85-139.

[2] Calderón A., Zygmund A., A note on the interpolation of sublinear operators., Amer. J. Math., 1956, 78, 282-288.

[3] Calderón A., Zygmund A., On singular integrals., Amer. J. Math., 1956, 78, 289-309.

[4] Calderón A., Zygmund A., A note on singular integrals., Studia Math., 1979, 65, 77-87.

[5] Hu G., Weighted vector-valued estimates for a non-standard Calderón-Zygmund operator., Nonlinear Analysis-Theory Methods and Applications, 2017, 165, 143-162. 
[6] He S., Zhou J., Vector-valued maximal multilinear Calderón-Zygmund operator with nonsmooth kernel on weighted Morrey space., Journal of Pseudo-differential Operators and Applications, 2017, 8, 213-239.

[7] Yabuta K., Generalizations of Calderón-Zygmund operators, Studia Mathematica, 1985, 82, 17-31.

[8] Lan J., Weak type endpoint estimates for multilinear $\theta$-type Calderón-Zygmund operators, Acta Mathematicae Applicatae Sinica, English Series. 2005, 21, 615-622.

[9] Xie R., Shu L., $L^{p}$ boundedness of for the maximal multilinear singular integral operator of $\theta$-type Calderón-Zygmund kernel, J. Sys. Sci. and Math. Scis., 2009, 29, 519-526.

[10] Xie R., Shu L., On multilinear commutators of $\theta$-type Calderón-Zygmund operators, Analysis in Theory and Applications, 2008, 24, 260-270.

[11] Quek T., Yang D. On weighted weak hardy spaces over $\mathbb{R}^{n}$, Acta Mathe. Sinica (English Series), 2000, 16, 141-160.

[12] Ri C., Zhang Z., Boundedness of $\theta$-type Calderón-Zygmund operators on Hardy spaces with non-doubling measures, Journal of inequalities and Applications. 2015, 323, 1-10.

[13] Ri C., Zhang Z., Boundedness of $\theta$-type Calderón-Zygmund operators on non-homogeneous metric measure spaces. Front. Math. China, 2016, 11(1):141-153.

[14] Wang H., Boundedness of $\theta$-type Calderón-Zygmund operators and commutators in the genralized weighted weak Morrey spaces, Journal of Function spaces, Volume 2016, Article ID 1309348, 18 pages.

[15] Kováčik O., Rákosník J., On sapaces $L^{p(x)}$ and $w^{k, p(x)}$, Czechoslovak Math, 1991, 41, 592-618.

[16] Acerbi E., Mingione G., Gradient estimates for a class of parabolic systems, Duke. Math. J., 2007, 136, 285-230.

[17] Acerbi E., Mingione G., Regularity results for stationary electrorheological fluids, Arch. Ration. Mech. Anal., 2002, 164, 213-259.

[18] Diening L., Ružička M., Calderón-Zygmund operators on generalized Lebesgue spaces $L^{p}$ and problem related to fluid dynamics, J. Reine. Angew. Math., 2003, 563, 197-220.

[19] Ružička M., Electrorheological Fluids: Modeling and Mathematical Theory, Lecture Notes in Math, 1748, Heidelberg, Germany: Spinger-Verlag, 2000.

[20] Zhikov V. V., Averaging of functionals of the calculus of variations and elasticity theory, Izv. Akad. Nauk SSSR. Ser. Mat., 1986, 50, 675-710 (in Russian).

[21] Cruz-Uribe D., Fiorenza A., Martell J. M., Pérez C., The boundedness of classical operators on variable $L^{p}$ spaces, Ann. Acad. Sci. Fenn. Math., 2006, 31, 239-264.

[22] Karlovich AY., Lerner AK., Commutators of singular integrals on generalized $L^{p}$ spaces with variable exponent, Publ. Mat., 2005, 49, 111-125.

[23] Tao S., Li L., Boundedness of Marcinkiewicz integrals and commutators on Morrey spaces with variable exponent, Chinese Journal of Contemporay Mathematics, 2016, 37, 53-68.

[24] Wang L., Tao S., Parameterized Littlewood-Paley operators and their commutators on Herz spaces with variable exponent, Turkish J. Math., 2016, 40, 122-145.

[25] Yang Y., Tao S., Singular integrals with variable kernel and fractional differentiation in homogeneous Morrey-Herz-type Hardy spaces with variable exponents, Open Mathematics, 2018, 16, 326-345.

[26] Wang L., Tao S., Boundedness of Littlewood-Paley operators and their commutators on Herz-Morrey spaces with variable exponent, J. Inequal. Appl., 2014, 227, 1-17.

[27] Tao S., Yang Y., $\theta$-Type C-Z operators and fractional differentiations on variable exponent Lebesgue spaces, Submitted.

[28] Izuki M., Herz and amalgam spaces with variable exponent, the Haar wavelets and greediess of the wavelet system, East. J. Approx., 2009, 15, 87-109.

[29] Izuki M., Vector-valued inequalities on Herz spaces and characterizations of Herz-Soblev spaces with variable exponent, Glasnik Mat., 2010, 45, 475-503.

[30] Izuki M., Boundedness of commutators on Herz spaces with variable exponent, Rend. Cir. Mat. Palermo., 2010, $59,199-213$.

[31] Izuki M., Boundedness of sublinear operators on Herz spaces with variable exponent and applications to wavelet characterization, Anal. Math., 2010, 36, 33-50.

[32] Wang H., Liu Z., The wavelet characterization of Herz-type Hardy spaces with variable exponent, Annals of Functional Analysis, 2012, 3, 128-141.

[33] Almeida A., Hasanov J. S., Maximal and potential operators in variable exponent Morrey spaces, Georgian Math, J., 2008, 15, 195-208.

[34] Lu S., Yang D., Hu G., Herz type spaces and their applications, Science Press(Beijing), 2008.

[35] Stein EM., Singular integrals and differentiability properties of functions, Princeton: Princeton Univ Press. $1970,151$.

[36] Dening L., Maximal functions on Musielak-Orlicz spaces and generalized Lebesgue spaces, Bull. Sci. Math., 2005, 129, 657-700.

[37] Devore R., Sharply R., Maximal function measuring smoothness, Mem Amer Math Soc., 1984, 47, 184-196.

[38] Ho K-P., The fractional integral operators on Morrey spaces with variable exponent on unbounded domains, Math. Inequal. Appl., 2013, 16, 363-373. 